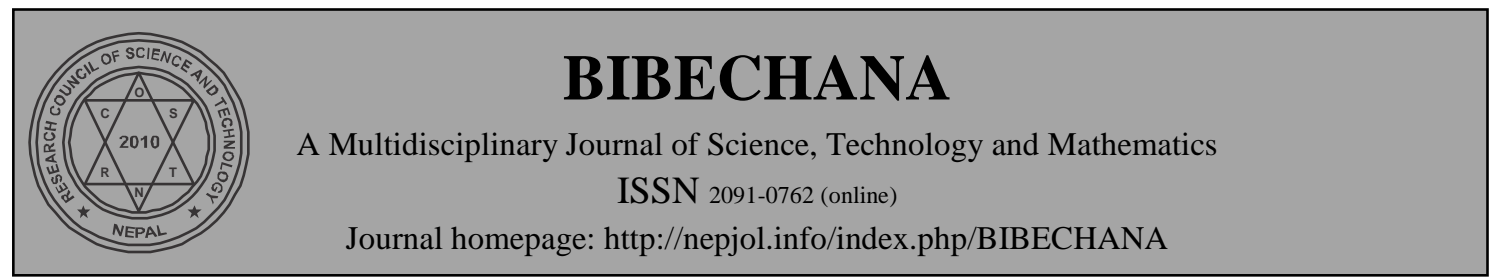

\title{
Stability of the Sitnikov's circular restricted three body problem when the primaries are oblate spheroid
}

\author{
R.R. Thapa \\ Department of Mathematics, Post Graduate Campus, Biratnagar \\ Tribhuvan University, Nepal \\ Email: thaparajuram@yahoo.com
}

Accepted for publication: February 06, 2014

\begin{abstract}
The Sitnikov's problem is a special case of restricted three body problem if the primaries are of equal masses $\left(m_{1}=\right.$ $m_{2}=1 / 2$ ) moving in circular orbits under Newtonian force of attraction and the third body of mass $m_{3}$ moves along the line perpendicular to plane of motion of primaries. Here oblate spheroid primaries are taken. The solution of the Sitnikov's circular restricted three body problem has been checked when the primaries are oblate spheroid. We observed that solution is depended on oblate parameter $A$ of the primaries and independent variable $\tau=\eta t$. For this the stability of non-trivial solutions with the characteristic equation is studied. The general equation of motion of the infinitesimal mass under mutual gravitational field of two oblate primaries are seen at equilibrium points. Then the stability of infinitesimal third body $\mathrm{m}_{3}$ has been calculated.
\end{abstract}

(C) 2014 RCOST: All rights reserved.

Keywords: Characteristic equation; Non-trivial solution; Oblate spheroid; Sitnikov's restricted three body problem; Stability.

\section{Introduction}

The primaries in the Sitnikov's problem move on the circumference of the same circle if the primaries are spheroid in shape. But if the primaries are oblate spheroid in shape then due to their oblateness the primaries will not be equidistant from their center of mass. The primaries should be equidistant from their centre of mass. For this Principal axes of the oblate bodies should be parallel to the synodic axes andThe masses of both the primaries should be equal.

The system consists of two oblate primaries with equal masses $\left(m_{1}=m_{2}=1 / 2\right)$. The third body of mass $\mathrm{m}_{3}$ is much less than the masses of the primaries. Thus the potential between two bodies $m_{1}$ and $m_{2}$ is given by

$$
\begin{aligned}
\Rightarrow-v & =\frac{G m_{1} m_{2}}{r}+\frac{G A\left(m_{1}+m_{2}\right)}{r^{3}} \\
A & =\frac{a^{2}-c^{2}}{10}=\text { oblatenless of the primaries }
\end{aligned}
$$

where $a_{i}, b_{i}, c_{i}(i=1,2)$ be the semi axes of the primaries. When the primaries are oblate, $a_{i}=b_{i}$.

An integrable case in restricted problem of three bodies by imposing further restrictions on the restricted three body problem, by supposing the two finite bodies of equal masses and an infinitesimal body moving in their common axis of revolution has been studied by MacMillan [1]. 
R.R. Thapa / BIBECHANA 11(1) (2014) 149-156: (Online Publication: March, 2014) p.150

In three body problem two primaries of equal masses and these two moving in (i) circular orbits and (ii) elliptic orbits around their center of mass has obtained by Sitnikov [2]. The third body (infinitesimal mass) is moving along a line which is perpendicular to the plane of primaries and passing through the center of mass of the primaries.

The stability and bifurcation of Sitnikov motion have studied by Perdios et.al. [3]. The family of periodic Sitnikov motions can be recognized as a special case $(\mu=1 / 2)$ of the family $L_{1}^{3}$ of three dimensional orbits emanating at $L_{1}$ for all admissible values of $\mu$ and is denoted by $L_{1}^{1}$. The existence of such a family offers the opportunity to study the stability of bifurcations of one-dimensional motions under perturbations in two additional dimensions which tends to take the moving particle directly into the full three-dimensional space.

The stability of motion in the Sitnikov problem have studied by Soulis et al. [4]. They have varied the mass of the infinitesimal body $0<m<10^{-3}$. They have found that as $\mathrm{m}_{3}$ increases, the domain of allowed motion grows significantly and chaotic regions in the phase space appear through a series of saddle-node bifurcations.

The equations of motions have the non-trivial solutions obtained by Mccuskey [5]

$$
\Rightarrow n^{2}=\frac{1}{r^{3}}+\frac{8 A}{r^{5}}
$$

The equation of motion of the third body has obtained by Suraj et al. [6], as follows

$$
\Rightarrow \frac{d^{2} z}{d t^{2}}+\frac{z}{\left(z^{2}+r^{2}\right)^{3 / 2}}+\frac{9 A z}{\left(z^{2}+r^{2}\right)^{5 / 2}}-\frac{15 A z^{2}}{\left(z^{2}+r^{2}\right)^{7 / 2}}=0
$$

\section{Solutions By Lindsted-Poincare Method}

Equation of motion of third body is

$$
\frac{d^{2} z}{d t^{2}}+\frac{z}{\left(z^{2}+r^{2}\right)^{3 / 2}}+\frac{9 A z}{\left(z^{2}+r^{2}\right)^{5 / 2}}-\frac{15 A z^{2}}{\left(z^{2}+r^{2}\right)^{7 / 2}}=0
$$

Raju Ram Thapa, et al. [7] has obtained the solution as follows:

$$
\begin{aligned}
& z=c \cos \eta t+\frac{3}{64}(\cos \eta t-\cos 3 \eta t) c^{3}+\frac{9 c^{5}}{4096}(23 \cos \eta t-24 \cos 3 \eta t+\cos 5 \eta t) \\
& +\frac{27 c^{7}}{8}\left[\frac{547}{32768} \cos \eta t-\frac{297}{16384} \cos 3 \eta t+\frac{3}{2048} \cos 5 \eta t-\frac{1}{32768} \cos 7 \eta t\right] \\
& +A\left[\frac{1}{32}(\cos \eta t-\cos 3 \eta t) c^{3}+\frac{3}{1024}(23 \cos \eta t-24 \cos 3 \eta t+\cos 5 \eta t) c^{5}\right. \\
& \left.+\frac{27}{4}\left(\frac{547}{32768} \cos \eta t-\frac{297}{16384} \cos 3 \eta t+\frac{3}{2048} \cos 5 \eta t-\frac{1}{32768} \cos 7 \eta t\right) c^{7}+\ldots\right]
\end{aligned}
$$

\section{Stability:}

The general equations of motion of the infinitesimal mass under the mutual gravitational field of two oblate primaries are 
R.R. Thapa / BIBECHANA 11(1) (2014) 149-156: (Online Publication: March, 2014) p.151

$$
\left.\begin{array}{r}
\ddot{x}-2 n \dot{y}=\frac{\partial \Omega}{\partial x} \\
\ddot{y}+2 n \dot{x}=\frac{\partial \Omega}{\partial y} \\
\ddot{z}=\frac{\partial \Omega}{\partial z}
\end{array}\right\}
$$

where $n$ is the mean angular velocity of the primaries moving on the common circular orbit of radius $a / 2$.

Here,

$$
\begin{aligned}
\Omega & =\frac{1}{\left(z^{2}+r^{2}\right)^{\frac{1}{2}}}+\frac{A}{\left(z^{2}+r^{2}\right)^{\frac{3}{2}}}-\frac{3 A z^{2}}{\left(z^{2}+r^{2}\right)^{\frac{5}{2}}} \\
b & =1+\frac{16}{3} A
\end{aligned}
$$

$$
\mathrm{A}=\text { oblateness parameter of the primaries. }
$$

Hence from (2), it is clear that $\Omega$ is independent of $x$ and $y$ and depends only upon $z$ alone hence $\frac{\partial \Omega}{\partial x}=\frac{\partial \Omega}{\partial y}=0$.

$$
\frac{\partial \Omega}{\partial z}=-\frac{z}{\left(z^{2}+b\right)^{\frac{3}{2}}}-\frac{9 A z}{\left(z^{2}+b\right)^{\frac{5}{2}}}+\frac{15 A z^{3}}{\left(z^{2}+b\right)^{\frac{7}{2}}}
$$

For the equilibrium solution of the restricted three body problem,

$$
\frac{\partial \Omega}{\partial x}=\frac{\partial \Omega}{\partial y}=\frac{\partial \Omega}{\partial z}=0
$$

Thus the system of equations (1) can be written as

$$
\left.\begin{array}{rl}
\ddot{x}-2 n \dot{y} & =\frac{\partial \Omega}{\partial x}=f(x, y, z) \text { (say) } \\
\ddot{y}+2 n \dot{x} & =\frac{\partial \Omega}{\partial y}=g(x, y, z) \text { (say) } \\
\ddot{z} & =\frac{\partial \Omega}{\partial z}=h(x, y, z) \text { (say) }
\end{array}\right\} \text { at the equilibrium points }
$$

Let us denote the equilibrium point as $L\left(0,0, z_{0}\right)$, we shall now communicate small displacement $\xi$, $\eta, \zeta$ in the coordinates of $L$ such that $x=0+\xi, y=0+\eta, z=z_{0}+\zeta$ satisfy the above equation. 
R.R. Thapa / BIBECHANA 11(1) (2014) 149-156: (Online Publication: March, 2014) p.152

Thus,

$$
\begin{gathered}
\Rightarrow \frac{d^{2} \xi}{d t^{2}}-2 n \dot{\eta}=f\left(0+\xi, 0+\eta, z_{0}+\zeta\right) \\
\frac{d^{2} \eta}{d t^{2}}-2 n \dot{\xi}=g\left(0+\xi, 0+\eta, z_{0}+\zeta\right) \\
\frac{d^{2} \zeta}{d t^{2}}=h\left(0+\xi, 0+\eta, z_{0}+\zeta\right) \text { where } \frac{d^{2} z_{0}}{d t^{2}}=0 . \\
\ddot{\xi}-2 n \dot{\eta}=f\left(0,0, z_{0}\right)+\xi\left(\frac{\partial f}{\partial x}\right)_{0}+\eta\left(\frac{\partial f}{\partial y}\right)_{0}+\zeta\left(\frac{\partial f}{\partial z}\right)_{0}+\text { higher order infinitesimals } \\
\ddot{\eta}+2 n \dot{\xi}=g\left(0,0, z_{0}\right)+\xi\left(\frac{\partial g}{\partial x}\right)_{0}+\eta\left(\frac{\partial g}{\partial y}\right)_{0}+\zeta\left(\frac{\partial g}{\partial z}\right)_{0}+\text { higher order infinitesimals } \\
\ddot{\zeta}=h\left(0,0, z_{0}\right)+\xi\left(\frac{\partial h}{\partial x}\right)_{0}+\eta\left(\frac{\partial h}{\partial y}\right)_{0}+\zeta\left(\frac{\partial h}{\partial z}\right)_{0}+\text { higher order infinitesimals }
\end{gathered}
$$

Since $\left(0,0, z_{0}\right)$ is a solution of the set of equation (4)

$$
\begin{aligned}
& \frac{\partial \Omega}{\partial x}=\frac{\partial \Omega}{\partial y}=\frac{\partial \Omega}{\partial z}=0, \text { hence } \\
& f\left(0,0, z_{0}\right)=g\left(0,0, z_{0}\right)=h\left(0,0, z_{0}\right)=0 \\
& \ddot{\xi}-2 n \dot{\eta}=\xi \frac{\partial}{\partial x}\left(\frac{\partial \Omega}{\partial x}\right)_{0}+\eta \frac{\partial}{\partial y}\left(\frac{\partial \Omega}{\partial x}\right)_{0}+\zeta \frac{\partial}{\partial z}\left(\frac{\partial \Omega}{\partial x}\right)_{0}+\text { higher order infinitesimals } \\
& \text { Thus, } \quad \ddot{\eta}+2 n \dot{\xi}=\xi \frac{\partial}{\partial x}\left(\frac{\partial \Omega}{\partial y}\right)_{0}+\eta \frac{\partial}{\partial y}\left(\frac{\partial \Omega}{\partial y}\right)_{0}+\zeta \frac{\partial}{\partial z}\left(\frac{\partial \Omega}{\partial y}\right)_{0}+\text { higher order infinitesimals } \\
& \ddot{\zeta}=\xi \frac{\partial}{\partial x}\left(\frac{\partial \Omega}{\partial z}\right)_{0}+\eta \frac{\partial}{\partial y}\left(\frac{\partial \Omega}{\partial z}\right)_{0}+\zeta \frac{\partial}{\partial z}\left(\frac{\partial \Omega}{\partial z}\right)_{0}+\text { higher order infinitesimals }
\end{aligned}
$$

Neglecting higher order terms of $\xi, \eta, \zeta$, we get the variational equations as

$$
\left.\begin{array}{r}
\Rightarrow \ddot{\xi}-2 n \dot{\eta}=\xi \Omega_{x x}^{0}+\eta \Omega_{y x}^{0}+\zeta \Omega_{z x}^{0} \\
\ddot{\eta}+2 n \dot{\xi}=\xi \Omega_{x y}^{0}+\eta \Omega_{y y}^{0}+\zeta \Omega_{z y}^{0} \\
\ddot{\zeta}=\xi \Omega_{x z}^{0}+\eta \Omega_{y z}^{0}+\zeta \Omega_{z z}^{0}
\end{array}\right\}
$$

where $\Omega_{x x}^{0}, \Omega_{x y}^{0}, \Omega_{x z}^{0}, \ldots$ etc represent the value of $\frac{\partial}{\partial x}\left(\frac{\partial \Omega}{\partial x}\right)_{0}, \frac{\partial}{\partial x}\left(\frac{\partial \Omega}{\partial y}\right)_{0}, \frac{\partial}{\partial x}\left(\frac{\partial \Omega}{\partial z}\right)_{0}, \ldots$ at the equilibrium points. 
R.R. Thapa / BIBECHANA 11(1) (2014) 149-156: (Online Publication: March, 2014) p.153

Now the system of equations (5) can be written in the form of a single matrix equation as

$$
\left[\begin{array}{c}
\dot{\xi} \\
\dot{\eta} \\
\dot{\zeta} \\
\ddot{\xi} \\
\ddot{\eta} \\
\ddot{\zeta}
\end{array}\right]_{6 \times 1}=\left[\begin{array}{cccccc}
0 & 0 & 0 & 1 & 0 & 0 \\
0 & 0 & 0 & 0 & 1 & 0 \\
0 & 0 & 0 & 0 & 0 & 1 \\
\Omega_{x x}^{0} & \Omega_{y x}^{0} & \Omega_{z x}^{0} & 0 & 2 n & 0 \\
\Omega_{x y}^{0} & \Omega_{y y}^{0} & \Omega_{z y}^{0} & -2 n & 0 & 0 \\
\Omega_{z x}^{0} & \Omega_{z y}^{0} & \Omega_{z z}^{0} & 0 & 0 & 0
\end{array}\right]_{6 \times 6}\left[\begin{array}{c}
\xi \\
\eta \\
\zeta \\
\dot{\xi} \\
\dot{\zeta}
\end{array}\right]_{6 \times 1}
$$

i.e. $\dot{X}=A X$

when $\left[\begin{array}{llllll}\xi & \eta & \zeta & \dot{\xi} & \dot{\eta} & \dot{\zeta}\end{array}\right]^{T}$ is a $6 \times 1$ column matrix of derivatives of the variations of the coordinates of the equilibrium points.

$$
A=\left[\begin{array}{cccccc}
0 & 0 & 0 & 1 & 0 & 0 \\
0 & 0 & 0 & 0 & 1 & 0 \\
0 & 0 & 0 & 0 & 0 & 1 \\
\Omega_{x x}^{0} & \Omega_{y x}^{0} & \Omega_{z x}^{0} & 0 & 2 n & 0 \\
\Omega_{x y}^{0} & \Omega_{y y}^{0} & \Omega_{z y}^{0} & -2 n & 0 & 0 \\
\Omega_{z x}^{0} & \Omega_{z y}^{0} & \Omega_{z z}^{0} & 0 & 0 & 0
\end{array}\right]_{6 \times 6}
$$

is a $6 \times 6$ square matrix of constants.

$$
\begin{aligned}
\text { But } \quad \begin{array}{l}
\Omega_{x x}^{0}=\Omega_{y x}^{0}=\Omega_{z x}^{0}=0 \\
\partial x
\end{array}=\frac{\partial \Omega}{\partial y}=0, \text { so } \begin{array}{l}
\Omega_{x y}^{0}=\Omega_{y y}^{0}=\Omega_{z y}^{0}=0 \\
\Omega_{z x}^{0}=\Omega_{z y}^{0}=0 \text { and } \Omega_{z z}^{0} \neq 0
\end{array} \\
A=\left[\begin{array}{ccccccc}
0 & 0 & 0 & 1 & 0 & 0 \\
0 & 0 & 0 & 0 & 1 & 0 \\
0 & 0 & 0 & 0 & 0 & 1 \\
0 & 0 & 0 & 0 & 2 n & 0 \\
0 & 0 & 0 & -2 n & 0 & 0 \\
0 & 0 & \Omega_{z z}^{0} & 0 & 0 & 0
\end{array}\right]
\end{aligned}
$$


R.R. Thapa / BIBECHANA 11(1) (2014) 149-156: (Online Publication: March, 2014) p.154

If any matrix $X$ satisfies the equation $A X=\lambda X$

The first in the solution of (7) is to found as the value of $X$.

We can write (8) as

$$
(A-\lambda I) X=0
$$

where $I$ is the $6 \times 6$ unit matrix.

The set of six simultaneous linear equations in six unknowns $\xi, \eta, \zeta, \dot{\xi}, \dot{\eta}, \dot{\zeta}$ will have non-trivial solutions provided the determinant of the matrix $(A-\lambda I)$ vanishes. i.e.

$$
|A-\lambda I|=0
$$

To check the stability of the (10) non-trivial solutions. Let us find values of $\lambda$ from (10) i.e. from

$$
\Rightarrow \lambda^{2}\left(\lambda^{2}+4 n^{2}\right)\left(\lambda^{2}-\Omega_{z z}^{0}\right)=0
$$

i.e. there are six values of $\lambda$. Let $\lambda_{1}^{2}, \lambda_{2}^{2}, \lambda_{3}^{2}$ be three roots corresponding each factor of (11). The conditions for stable solution are

$$
\lambda_{i}^{2} \leq 0 \quad \text { for } i=1,2,3 \text {. }
$$

From (11)

$$
\left.\begin{array}{c}
\lambda_{1}^{2}=0 \Rightarrow \lambda_{11}=\lambda_{12}=0 \\
\Rightarrow \lambda_{2}= \pm 2 n i \text { (imaginary) } \\
\Rightarrow \lambda_{21}=2 n i, \quad \lambda_{22}=-2 n i \\
\text { when } \lambda_{3}^{2}-\Omega_{z z}^{0}=0 \Rightarrow \lambda_{3}= \pm \sqrt{\Omega_{z z}^{0}}
\end{array}\right\}
$$

$$
\begin{aligned}
& \text { But } \quad \frac{\partial \Omega}{\partial z}=-\frac{z}{\left(z^{2}+b^{2}\right)^{\frac{3}{2}}}-\frac{9 A z}{\left(z^{2}+b^{2}\right)^{\frac{5}{2}}}+\frac{15 A z^{3}}{\left(z^{2}+b^{2}\right)^{\frac{7}{2}}} \\
& \Rightarrow \frac{\partial^{2} \Omega}{\partial z^{2}}=-\frac{z}{\left(z^{2}+b^{2}\right)^{\frac{3}{2}}}+\frac{3 z^{2}}{\left(z^{2}+b^{2}\right)^{\frac{5}{2}}}-\frac{9 A}{\left(z^{2}+b^{2}\right)^{\frac{5}{2}}}+\frac{90 A z^{2}}{\left(z^{2}+b^{2}\right)^{\frac{7}{2}}}-\frac{105 A z^{4}}{\left(z^{2}+b^{2}\right)^{\frac{9}{2}}}
\end{aligned}
$$




$$
\begin{aligned}
\Omega_{z z}^{0} & =-\frac{1}{b^{3}}\left(1-\frac{3}{2}\left(\frac{z_{0}}{b}\right)^{2}+\ldots\right)+\frac{3}{b^{3}}\left(\frac{z_{0}}{b}\right)^{2}\left(1-\frac{5}{2}\left(\frac{z_{0}}{b}\right)^{2}+\ldots\right)-\frac{9 A}{b^{5}}\left(1-\frac{5}{2}\left(\frac{z_{0}}{b}\right)^{2}+\ldots\right) \\
& +\frac{90 A}{b^{5}}\left(\frac{z_{0}}{b}\right)^{2}\left(1-\frac{7}{2}\left(\frac{z_{0}}{b}\right)^{2}+\ldots\right)-\frac{105 A}{b^{5}}\left(\frac{z_{0}}{b}\right)^{4}\left(1-\frac{9}{2}\left(\frac{z_{0}}{b}\right)^{2}+\ldots\right)
\end{aligned}
$$

Since $z_{0}$ is very small in comparison of $\mathrm{b}$ hence $\frac{z_{0}}{b}<<1$ and so $\left(\frac{z_{0}}{b}\right)^{2},\left(\frac{z_{0}}{b}\right)^{3},\left(\frac{z_{0}}{b}\right)^{4}, \ldots$ are negligible.

Thus we get

$$
\begin{aligned}
\Omega_{z z}^{0} & =-\frac{1}{b^{3}}-\frac{9 A}{b^{5}}=-\left[\frac{1}{\left(1+\frac{16}{3} A\right)^{3}}+\frac{9 A}{\left(1+\frac{16}{3} A\right)^{5}}\right] \\
& =-\left[\left(1+\frac{16}{3} A\right)^{-3}+9 A\left(1+\frac{16}{3} A\right)^{-5}\right] \mathrm{using} r^{2}=1+\frac{6 \mathrm{~A}}{3}=b \\
& =-[1+A] \\
& \Omega_{z z}^{0}=-(1+A) \\
\Rightarrow & \lambda_{3}^{2}=-(1+A) \\
\Rightarrow & \lambda_{3}= \pm i \sqrt{1+A} \\
\Rightarrow & \lambda_{31}=i \sqrt{1+A} ; \quad \lambda_{32}=-i \sqrt{1+A}
\end{aligned}
$$

Thus, all the six roots of the characteristic equation are 0,$0 ; 2 \mathrm{ni},-2 \mathrm{ni}, i \sqrt{1+A} ;-i \sqrt{1+A}$ and hence the equilibrium position of the Sitnikov restricted three body problem are stable.

\section{Conclusion}

The condition of stability is satisfied by solution of the Sitnikov's problem the third body is oscillatory in nature. It displaced slightly from its equilibrium position but stayed in the neighbourhood of the equilibrium point. 
R.R. Thapa / BIBECHANA 11(1) (2014) 149-156: (Online Publication: March, 2014) p.156

\section{Acknowledgments}

The author is grateful to his respected Supervisor Dr. M.R. Hassan, Dept. of Mathematics, S.M. College, Bhagalpur, T.M.B. University, Bhagalpur, 812007, India and also would like to gratefully acknowledge Prof. Dr. Devendra Adhikari (P.G. Department of Physics, M.M.A.M. Campus, Biratnagar, Nepal) for inspiring discussion.

\section{References}

[1] W.D. Macmillan, Astronomical Journal, 27 (1913) 11,

[2] K.A. Stinikov, Dokl. Akad. Nauk, USSR, 133 (1960) 303.

[3] E. Perdois, et al., Celest. Mech. \& Dyn. Astron., 42 (1988) 187.

[4] P.S. Soulis, Celest. Mech. \& Dyn. Astron., 99 (2007) 129.

[5] S.W. Mccuskey Introduction to Cel. Mechanics, Addison-Wesley publishing company, Inc., 1963.

[6] M.S. Suraj, M.R.Hassan, Proceeding of PAS, 50 (2013) 61.

[7] R.R. Thapa, M.R. Hassan, Proceedings: Modern Trends in Science and Technology, Biratnagar (2013) 129. 